\title{
La Evolución Histórica de los Elementos Nocivos en el Medio Ambiente de Madrid
}

\author{
Salama Saleima Omar ${ }^{1}$
}

\begin{abstract}
Resumen
En este trabajo se aborda la influencia de la contaminación ambiental en el globo terráqueo, desde los primeros años de la revolución industrial hasta nuestros días. Además se realiza un análisis de los elementos nocivos y su evolución en la ciudad de Madrid. También se revisa el cumplimento de los tratados de la comunidad europea y España, para reducir dicha contaminación.
\end{abstract}

Palabras-clave: Contaminación. Medio ambiente. Isla de calor. Calidad del aire.

\section{THE HISTORICAL EVOLUTION OF THE HARMFUL ELEMENTS IN THE ENVIRONMENT OF MADRID.}

\begin{abstract}
In this work is addressed the influence of environmental pollution in the globe earth, from the early years of the industrial revolution until today. In addition an analysis of the harmful elements and their evolution in the city of Madrid is made. The compliance with the treaties of the European Community and Spain, to reduce such pollution is also reviewed.
\end{abstract}

Keywords: Pollution, environment, heat island, air quality.

1 Doctor en Ciencias Químicas. Investigador contratado en Ingeniería Química. Universidad Autónoma de Madrid, Departamento de Química Física Aplicada. salama.omar@uam.es 
Desde finales del siglo 16 con la gran aparición de la revolución industrial y el paulatino aumento de la contaminación generada en las grandes ciudades trae consigo el debate entre el crecimiento económico y la conservación del medio ambiente. Durante el siglo pasado, el crecimiento económico, basado en la explotación desmesurada de los recursos naturales básicos de la tierra que eran considerados de escaso valor e importancia, por su nuevo uso, condujo a transformaciones en la vida del campo y a constantes fluctuaciones poblacionales hacia las grandes ciudades que se han ido creando a través de procesos de urbanización sin control y se caracterizaron por el desempleo, la inseguridad, la pobreza y la infravivienda. Por consiguiente, estas migraciones crearon una urbanización desordenada entorno a las áreas urbanas. Dicha explotación sin límites de los recursos naturales y su aumento en el consumo condujo a diversas transformaciones en la vida de los bosques y zonas rurales. Además, se produjo una paulatina sobreexplotación al límite de los recursos naturales y un aumento incontrolado en el consumo de estos recursos naturales, como las materias primas, energía, bosques, recursos hídricos y biodiversidad y tierra fértil, forman la base de nuestra existencia en el planeta tierra. Como por ejemplo, nuestra vestimenta, la comida que consumimos, el agua y todas demás cosas que utilizamos a diario, son fabricados con recursos naturales que se extraen de la tierra y se transforman por el ser humano. Sin embargo, el rápido crecimiento del consumo de estos recursos por parte de la humanidad está causando daños considerables. Nuestro clima está cambiando; las reservas de agua dulce, los recursos pesqueros y los bosques están en peligro; las tierras fértiles se está desgastando y cada vez son más las especies que se extinguen. Por lo que, para que podamos seguir prosperando, nuestro estilo de vida tiene que volverse más sostenible con el fin de poder proteger nuestra base de recursos naturales y los frágiles ecosistemas.

Dichos asentamientos periurbanos han contribuido a la destrucción sistemática de los entornos naturales y de los valiosos ecosistemas naturales al mismo tiempo que propiciaron la continua producción de tecnología para defender la calidad de vida que se vislumbraba permanentemente amenazada por la naturaleza. Por lo que no se tenían en cuenta las consecuencias ambientales en las etapas iniciales del auge industrial, donde la gran ambición desmesurada 
del crecimiento económico, por mejorar las condiciones de vida podría causar modificaciones irreversibles en nuestro planeta, que afectarían a las generaciones venideras. Como por ejemplo, se destruyen montañas para extraer minerales, se talan bosques para conseguir madera, se sobreexplotan los acuíferos, se extinguen especies animales, se llena el aire de gases tóxicos, etc. Por lo que, los conocimientos científicos muestran que los recursos naturales no son ilimitados y que el desarrollo y la organización de la industria orientada al mercado. Las prácticas agrícolas masivas, la generación de residuos y contaminantes difícilmente soportables por los ecosistemas, la obtención y uso desproporcionado de la energía y los hábitos de vida y de consumo están perjudicando gravemente los ecosistemas y poniendo en grave peligro la vida en el planeta y la supervivencia de los seres vivos. Además, el transporte, es uno de los principales causante de la contaminación de la atmósfera, de modo que genera la tercera parte del monóxido de carbono, la mitad de los hidrocarburos, óxidos de nitrógeno y plomo, y la cuarta parte de las partículas (LÓPEZ BONILLA, 1994). Además, esta actividad terciaria supone un factor clave en la alteración de la composición normal del aire.

Siendo todo esto, se le conoce como contaminación medio ambiental. Donde, las causas de estos problemas ambientales están arraigadas en la conducta humana. Visto lo anterior mencionado, la solución podría encontrarse en el cambio de la conducta de los grupos y de las organizaciones y en las conductas políticas de control del crecimiento de la población y del uso eficaz y controlado de los recursos y, por tanto, en cambios culturales y de estilos de vida.

\section{Tipos de contaminación en las ciudades}

Las causas de la contaminación ambiental son variadas y dependen de la presencia en el ambiente de cualquier agente físico, químico o biológico, donde su concentración ocasione variaciones en la estructura molecular y el funcionamiento de los ecosistemas se considera como parte de este tipo de contaminación. Dentro de los contaminantes físicos encontramos la radioactividad, el calor, el ruido, efectos mecánicos, etc. Mientras dentro de los contaminantes químicos, 
que provienen principalmente de la industria química, encontramos los productos tóxicos minerales, ácidos, disolventes orgánicos, detergentes, plásticos, los derivados del petróleo, pesticidas, abonos sintéticos, etc.

Por los que, aquí podemos clasificar entre tres tipos de contaminación ambiental y sus causantes en: La contaminación del aire (o atmosférica) es la provocada por los humos de los vehículos e industrias, aerosoles, polvo, ruidos, malos olores, radiación atómica, etc. Entre los contaminantes gaseosos más comunes encontramos, el dióxido de carbono $\left(\mathrm{CO}_{2}\right)$, el óxido de carbono, los hidrocarburos, los óxidos de nitrógeno, los compuestos orgánicos volátiles (COVs), los óxidos de azufre y el ozono. Por lo que, las principales causas de la contaminación del aire están relacionadas con la quema de combustibles fósiles (carbón, petróleo y gas). La combustión de estas materias primas se produce en los procesos o en el funcionamiento de los sectores industrial y del transporte por carretera, principalmente. Según datos de Ecologistas en Acción, aproximadamente el 80\% de la contaminación atmosférica en España está causada por el transporte frente a la industria, ya sea la de fabricación o la de producción eléctrica. ${ }^{2}$

\section{Cronología de algunos de los contaminantes}

Por lo tanto, en este trabajo, se describe una breve reseña sobre algunos de los contaminantes, por ejemplo, el dióxido de carbono $\left(\mathrm{CO}_{2}\right)$ : es un gas que se forma en la combustión de todo combustible, por oxidación de los átomos de carbono. Siendo su fuente principal son, los organismos vivos que lo emiten como producto final de la oxidación de azúcares y otros compuestos orgánicos que contienen carbono. Su emisión, se debe fundamentalmente a los procesos que generan energía, de tipo eléctrica como de calefacción y otros en instalaciones industriales, así como en los vehículos de transporte y en plantas de tratamientos de residuos, etc.

2 Disponible en: $<$ http://www.ecologistasenaccion.es/>. 
Por ser un gas bastante estable, su evolución en la atmósfera, es a través de que, puede disolverse en agua formando ácido carbónico que, en situaciones normales, es el compuesto promotor del $\mathrm{pH}$ ligeramente ácido del agua de lluvia y además es uno de los gases causantes del efecto invernadero. El incremento de su concentración en la atmósfera está reforzando dicho efecto. Fundamentalmente, el aumento del efecto invernadero produce una afección sobre el clima, alterando el equilibrio de radiación, dado que permite el paso de la radiación solar, pero absorbe la radiación infrarroja emitida por la Tierra. El consecuente incremento en la temperatura atmosférica podría derivar en alteraciones en las corrientes marinas a gran escala, interconectadas con posibles deshielos polares, especialmente en el Ártico y, por consiguiente, en una variación en los regímenes de lluvias de amplias regiones, lo que podría derivar finalmente en una modificación ambiental del ecosistema y una negativa repercusión sobre la producción de alimentos.

Mientras que otro contaminante gaseoso, como los óxidos de nitrógeno (el término $\mathrm{NO}_{x}$, se refiere a la combinación de ambas sustancias: $\mathrm{NO}, \mathrm{NO}_{2}$ ), Se ha hecho referencia a las propiedades, efectos sobre la salud y el medio ambiente del dióxido de nitrógeno, ya que es el contaminante principal de los $\mathrm{NO}_{\mathrm{X}}$.

El dióxido de nitrógeno es el principal contaminante de los óxidos de nitrógeno, y se forma como subproducto en todas las combustiones llevadas a cabo a altas temperaturas. Se trata de una sustancia de color amarillento, que se forma en los procesos de combustión en los vehículos motorizados y las plantas eléctricas. Por lo que, es un gas tóxico, irritante y precursor de la formación de partículas de nitrato, que conllevan la producción de ácidos y elevados niveles de $\mathrm{PM}_{2.5}$ en el ambiente. Presenta buena solubilidad en agua, dando lugar al ácido nítrico $\left(\mathrm{HNO}_{3}\right)$ según, se describe en la siguiente reacción: $\mathrm{NO}_{2}+\mathrm{H}_{2} \mathrm{O}->$ $2 \mathrm{HNO}_{3}+\mathrm{NO}$. Esta sustancia es un oxidante fuerte y reacciona violentamente con materiales combustibles y reductores, pudiendo atacar materiales metálicos en presencia de agua. También es producto de la descomposición bacteriana de nitratos orgánicos, los incendios forestales, quema de rastrojos y la actividad volcánica. Además es producto de la emisión, que se producen en los escapes de los vehículos motorizados y en la quema de combustibles fósiles. También, 
pero en menor relevancia, se llevan a cabo en los procesos biológicos de los suelos, en los que se produce la emisión de nitritos $\left(\mathrm{NO}_{2}\right)$, por parte de los microorganismos.

La inhalación de estos contaminantes atmosféricos como el ozono, los metales y los radicales libres, en elevadas concentraciones y durante un corto periodo de tiempo, puede originar un edema pulmonar cuyos efectos no se observan hasta pasadas unas horas, agravándose con el esfuerzo físico. Una exposición prolongada puede afectar al sistema inmune y al pulmón, dando lugar a una menor resistencia frente a infecciones y causa cambios irreversibles en el tejido pulmonar.

Por otra parte el $\mathrm{NO}_{2}$, se forma a partir de la oxidación del óxido nítrico (NO), y tiene una vida corta en la atmósfera ya que se oxida rápidamente a nitratos $\left(\mathrm{NO}_{3}\right)$ o a $\mathrm{HNO}_{3}$ (ácido nítrico). En este último caso, se produce el fenómeno de la lluvia ácida que consiste en la reacción de los nitratos $\left(\mathrm{NO}_{3}\right)$ con la humedad existente en el ambiente, dando lugar a ácido nítrico $\left(\mathrm{HNO}_{3}\right)$, que precipita causando grandes destrozos en los bosques y la acidificación de las aguas superficiales.

Los compuestos orgánicos volátiles (COVs), son compuestos orgánicos constituidos fundamentalmente por carbono, que se convierten fácilmente en vapor o gas. De forma general, presentan puntos de ebullición que oscilan entre 50 y $260^{\circ} \mathrm{C}$, (WHO, 2000a, b; GUENTHER, 1995; RUDD, 1995). Los heteroátomos más habituales que forman parte de los COVs suelen ser oxígeno, flúor, cloro, bromo, azufre o nitrógeno. Estos compuestos agrupan a una gran cantidad de tipos de compuestos químicos, entre los que se incluyen los hidrocarburos alifáticos y aromáticos (clorados o no), y otros compuestos como aldehídos, cetonas, éteres, ácidos y alcoholes. Los COVs, son liberados por la quema de combustibles, como gasolina, madera, carbón o gas natural. También son liberados por disolventes, pinturas, pegamentos y otros productos empleados y almacenados en los hogares y centros de trabajo. Al ser liberados al aire tienen la capacidad para formar ozono en relación a etileno también denominado factor 
de producción de ozono troposférico (POCP, Photochemical Ozone Creation Potential). Es importante señalar el control de la incidencia en el Medio Ambiente por el concepto $\mathrm{POCP}\left[\mathrm{NO}+\mathrm{O}_{3}=\mathrm{NO}_{2}+\mathrm{O}_{2}\right]$.

Siendo la mayoría de estos compuestos orgánicos volátiles son peligrosos contaminantes del aire. Con respecto a los impactos producidos en el medio ambiente, se trata de unas sustancias que tienen una gran trascendencia en la formación del smog fotoquímico, ya que al combinarse con otros contaminantes atmosféricos por ejemplo los compuestos orgánicos volátiles de metano (COVDM) influyen en las reacciones de formación de ozono en la superficie de la tierra. Al mezclarse con óxidos de nitrógeno, reaccionan para dar lugar a: ozono, en el nivel del suelo o «smog» (de smoke: humo y fog: niebla). Este término se usa para designar la contaminación atmosférica que se produce en algunas ciudades como resultado de la combinación de unas determinadas circunstancias climatológicas y unos concretos contaminantes. Hay dos tipos muy diferentes de smog:

El llamado smog industrial o gris fue muy típico en algunas ciudades grandes, como Londres o Chicago, con mucha industria, en las que, hasta hace unos años, se quemaban grandes cantidades de carbón y petróleo pesado con mucho azufre, en instalaciones industriales y de calefacción (WICHMANN, 1985). En estas ciudades se formaba una mezcla de dióxido de azufre, gotitas de ácido sulfúrico formado a partir del anterior y una gran variedad de partículas sólidas en suspensión, que originaba una espesa niebla cargada de contaminantes, con efectos muy nocivos para la salud de las personas y para la conservación de edificios y materiales (FENGER, 1999).

De donde en muchas ciudades el principal problema de contaminación, es el llamado smog fotoquímico. Ya que con este nombre se refiere a una mezcla de contaminantes de origen primario (NOx e hidrocarburos volátiles) con otros secundarios (ozono, peroxiacilo, radicales hidroxilo, etc.) que se forman por reacciones producidas por la luz solar al incidir sobre los primeros. Esta mezcla oscurece la atmósfera dejando un aire teñido de color marrón rojizo cargado de componentes perjudiciales para los seres vivos (WHITTAKER et al., 2004). Aunque prácticamente en todas las ciudades del mundo hay problemas con este 
tipo de contaminación, es especialmente importante en las que están en lugares con clima seco, cálido y soleado, y con muchos vehículos. Siendo en la época de la estación del verano es la peor estación para este tipo de polución y, además, algunos fenómenos climatológicos, como las inversiones térmicas, pueden agravar este problema en determinadas épocas ya que dificultan la renovación del aire y la eliminación de los contaminantes. En la Figura 1 (NASA'S..., 2016) podemos ver el efecto que producen los incendios forestales recientemente en las nubes sobre el cielo de Canadá, donde las cenizas forman una estela a favor de la dinámica general atmosférica. Así, en este caso, las partículas, arrastradas por el viento húmedo, permiten reconocer el sentido de la borrasca en el hemisferio norte.

\section{Figura 1 - Efecto que producen los incendios forestales}

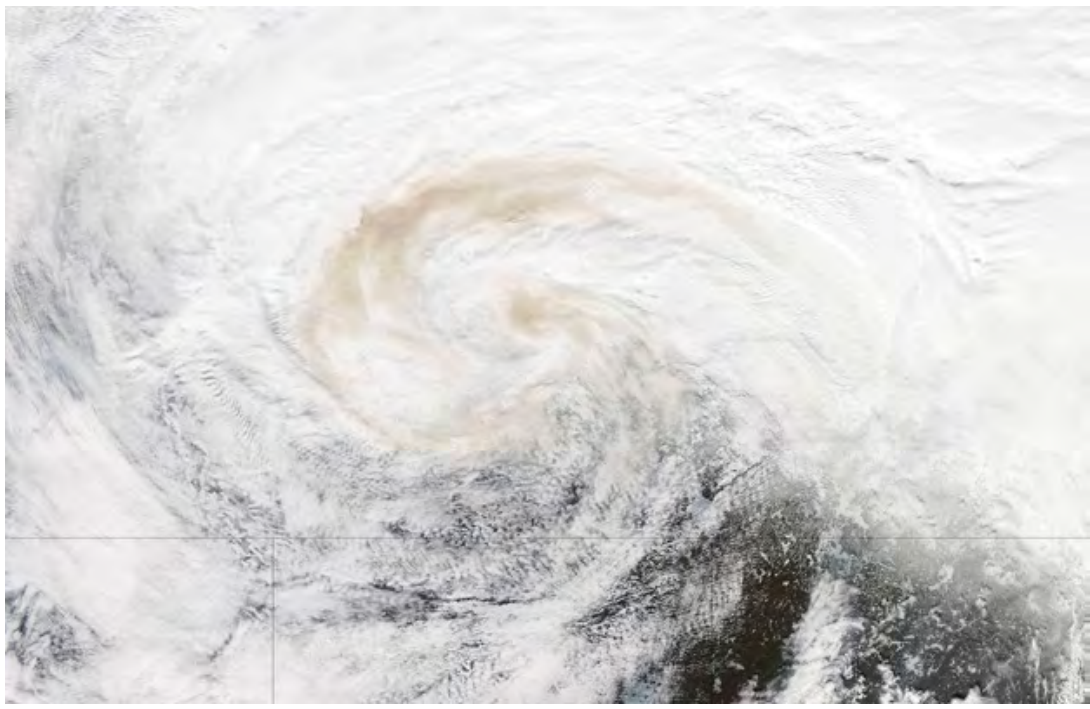

Fuente: NASA's, 2016.

Por ende, la creciente preocupación acerca de los efectos sobre la sostenibilidad del medio ambiente, el desarrollo social del modo de vida moderno, las conquistas de la tecnología y la producción industrial, es evidente en todos los países y en sectores cada vez más amplios de la población a través de los llamados movimientos verdes o ambientalista, de la actitud de la opinión pública 
y de las legislaciones de los estados que poco a poco comienzan a tomar más conciencia sobre los graves efectos de éstos elementos nocivos sobre nuestra salud y nuestro planeta tierra. Ya que los contaminantes nocivos gaseosos anteriormente mencionados, presentan una gran capacidad de penetración en las vías respiratorias y por ende existen en las ciudades indicadores que miden la contaminación urbana, de la concentración de las partículas materiales en el aire. Donde los valores de las partículas de diámetro inferior a 2,5 micras, $\mathrm{PM}_{2,5}$, parecen ser un mejor indicador de la contaminación urbana que las que se venían utilizando hasta ahora, las $\mathrm{PM}_{10}$. De hecho las $\mathrm{PM}_{2,5}$, en buena medida, son producto de las emisiones de los vehículos diésel en las ciudades. Mientras que las partículas de mayor tamaño pueden tener en su composición un importante componente de tipo natural, como partículas de polvo procedente de las intrusiones de viento del norte de África (polvo sahariano), frecuente en nuestras latitudes.

Figura 2 - Clasificación del material particulado en la atmósfera urbana
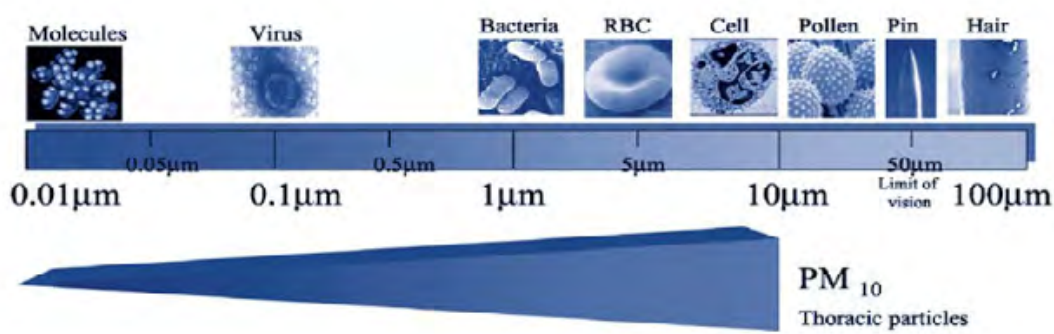

$\mathrm{PM}_{10}$

Thoracic particles

$\mathrm{PM}_{10-2.5}$

Coarse fraction

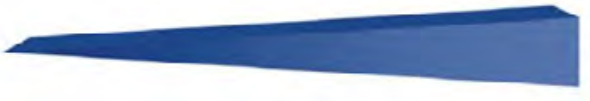

$\mathrm{PM}_{2.5}$

Fine particles

\section{UFP (PM $\left.{ }_{0.1}\right)$}

Ultrafine particles

Fuente: Ecologistas en Acción.

Por lo que Linares Gil y Díaz Jiménez (2008) han estudiado el comportamiento de estas partículas $\mathrm{PM}_{2,5}$ para la ciudad de Madrid y su impacto en la salud a través de su influencia sobre los ingresos hospitalarios. Este material 
respirable se encuentra en la atmósfera de nuestras ciudades en forma sólida o líquida (polvo, cenizas, hollín, partículas metálicas, cemento y polen, entre otras) se puede dividir, según su tamaño, en dos grupos principales. A las de diámetro aerodinámico igual o inferior a los $10 \mu \mathrm{m}$ o 10 micrómetros $(1 \mu \mathrm{m}$ corresponde a la milésima parte de un milímetro) se las denomina $\mathrm{PM}_{10}$ y a la fracción respirable más pequeña, $\mathrm{PM}_{2,5}$ (ver Figura 2).

\section{Tratados, Europeos, estatales}

En el ámbito de la Unión Europea (EEA, 2001; 2002; WHO, 2000a, b, $2002,2004)$ se han desarrollado muchos de los principios ya sentados por Naciones Unidas en el ámbito de la protección universal del medio ambiente. Es evidente que todos estos principios forman parte de la estrategia en conjunto de todos los estados miembros, para prevenir los posibles daños al medio ambiente y aplicar las sanciones impertinentes a sus estados miembros que violan la normativa vigente, para que se pueda reducir los riesgos del medio ambiente. Por consiguiente, los organismos internacionales son conscientes de la necesidad de establecer políticas de prevención ya que los procedimientos sancionadores, por sí mismos, no garantizan el cumplimiento de dichas normas. En la práctica de la UE, son los Estados los que, a través de las normas internas que desarrollan los postulados de las directivas comunitarias, establecen el marco normativo dentro del cual los distintos actores y agentes (empresas, industrias, centrales energéticos, particulares, etc.) deben desarrollar sus actividades. Siendo, el objetivo es aplicar unos parámetros de medición de la calidad del aire, aplicables a un determinado espacio en concreto, como por ejemplo, un día a partir de la cual, la información obtenida a través de los aparatos de medición y vigilancia de la contaminación del aire y estos son fijados según la legislación vigente. Los elementos contaminantes peligrosos para la salud de referencia son el $\mathrm{SO}_{2}$, CO, PM10, $\mathrm{O}_{3}$ y el NO , (EEA, 2001). 
Según la legislación Europea (EEA, 2001a, b; WHO, 2000a, b, 2002, 2004) y el Real decreto 1073/2002, del estado Español, es permitido un valor límite en el año, no superar más de 35 días al año el valor límite diario $(50 \mu \mathrm{g} /$ $\mathrm{m} 3$ en $24 \mathrm{~h}$ ), con fecha vigor de cumplimiento en enero de 2005. Donde se ha visto que hay problemas frecuentes en España y también en muchos países del sur de Europa, en su cumplimiento. Siendo Madrid, la capital de España, posee una alta densidad de vehículos y es influenciada, en algunas ocasiones, por el aporte de polvo sahariano. Por lo que se ha podido saber, que no ha sido posible el cumplimiento del valor límite acordado en las condiciones actuales, según los autores del estudio (Fuente: Ecologistas en Acción) y como se puede observar en la gráfica 3.

La vigilancia de la calidad del aire lleva realizándose en la ciudad de Madrid desde hace décadas, al igual que en el resto de ciudades europeas, en virtud de la directiva del Consejo Europeo 96/62/CE, y donde se adopta un planteamiento general sobre la evaluación de la calidad del aire (Comisión Europea, 1996), de donde sufrido varias modificaciones en numerosas ocasiones. Últimamente ha sido sustituida por una nueva, extendiéndose la vigilancia a nuevos contaminantes y ampliando los requisitos para realizar estas mediciones (Comisión Europea, 2008). Esta última directiva ha sido recientemente traslada al marco normativo español (Gobierno de España, 2011), si bien su aplicación comenzó un año antes en ciudades como Madrid. En ella se establecen los criterios para la correcta ubicación y registro de las estaciones, provocando el traslado de varias de éstas. 
Figura 3 - Superaciones en número de días de los valores límite diarios de $\mathrm{PM}_{10}$ en Madrid correspondiente a los años 2001-2007

\section{GRÁfica2. SuPERACIONES (EN No dIAS) dE LOS VALORES LÍMITE DIARIOS DE PM ${ }_{10}$ EN MAdRID (2001-2007). Fuente: Ecologistas en Acción}

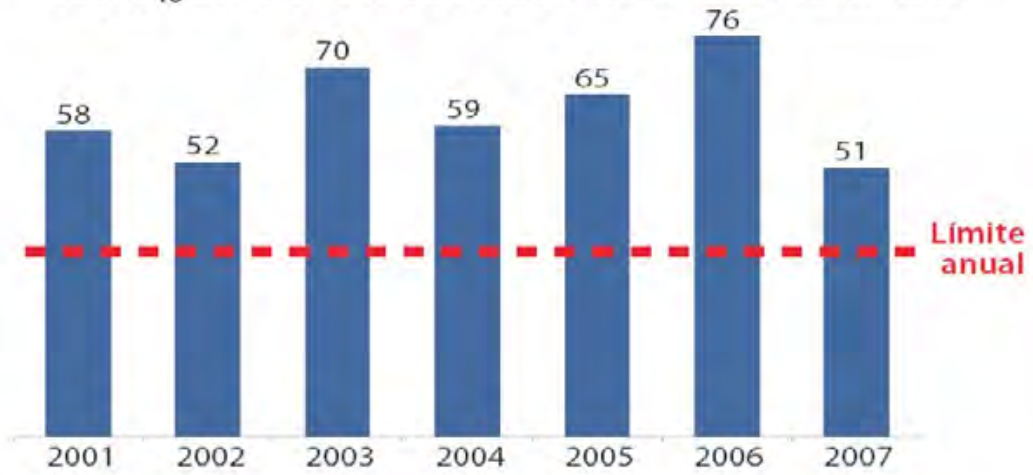

Fuente: Ecologistas en Acción.

De donde el Sistema Integral de la Calidad del Aire de Madrid (Sicam), encargado de los registros de la evolución de la contaminación, se encuentra distribuida por distintos pontos de la ciudad de Madrid, y todas sus estaciones se encuentran ubicadas a pocos metros por encima del nivel del suelo.

Según ecologistas en Acción el aire el aire de Madrid infringe sistemáticamente el valor límite permitido de dióxido de nitrógeno, $\mathrm{NO}_{2}$ y es establecido en $200, \mu \mathrm{g} / \mathrm{m}^{3}$, un valor que no debería rebasarse más de 18 horas al año. Por lo que es superado con mucha frecuencia durante los episodios de picos de contaminación (conocido como: la boina) que se producen recurrentemente en la ciudad. Episodios que tienen lugar bajo la influencia de factores meteorológicos (anticiclones con fenómenos de inversión térmica que limitan la dispersión de los contaminantes), aunque obviamente, el origen último de la contaminación no es la meteorología, sino el denso tráfico que sufre la ciudad diariamente y es fundamentalmente el de los vehículos diésel, que son más contaminantes. 
Figura 4 - Ilustra la Isla de Calor: en color rojo se puede observar las islas de calor más notables (Madrid y Barcelona), del territorio Español.

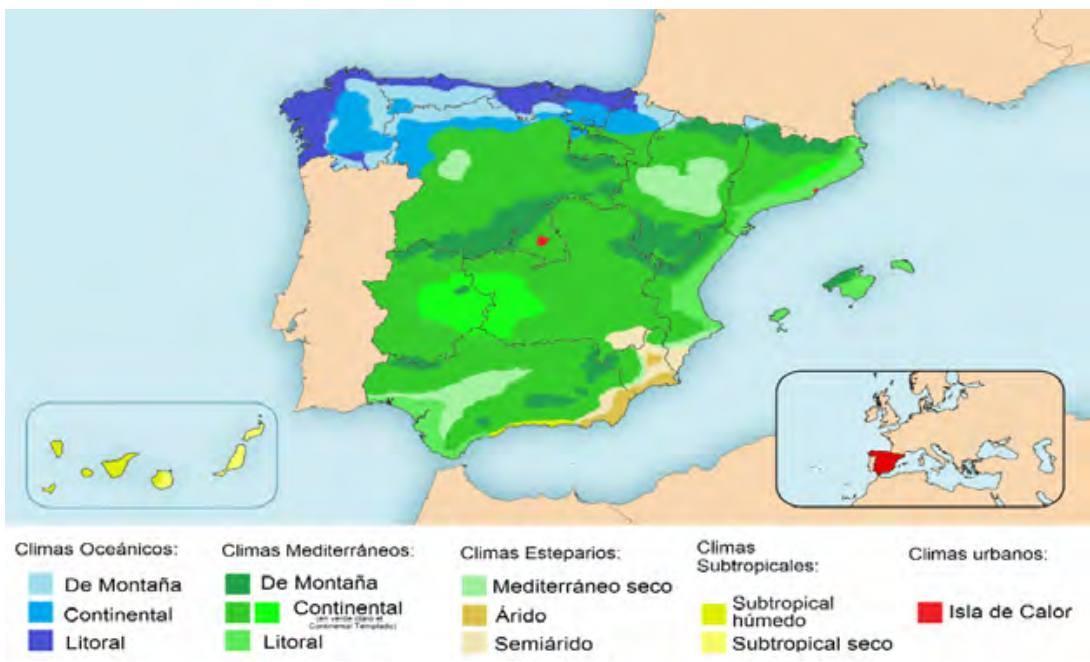

Fuente: Clima de la Península Ibérica.

Además, se ha observado que los gases contaminantes generan calor en las ciudades superpobladas y se conoce con el nombre de Islas de Calor (FERNÁNDEZ-GARCIA, 2012). Por lo que el clima, en particular el viento y las nubes, influyen en la formación de dicho fenómeno. Siendo sus magnitudes son mayores bajo condiciones climatológicas calmadas y claras. Ya que a medida que los vientos aumentan, se mezclan con aire y reducen la isla de calor. A medida que las nubes aumentan reducen el enfriamiento nocturno por radiación, y también reducen la isla de calor. Las variaciones estacionales de los patrones climáticos afectan la frecuencia y la magnitud de la isla de calor. También la localización geográfica influye sobre el clima y la topografía de la zona, así como sobre las características de las zonas rurales de la ciudad. Las influencias climáticas regionales o locales, tales como los sistemas locales de vientos, pueden afectar las islas de calor; por ejemplo, las ciudades costeras pueden experimentar un enfriamiento de las temperaturas urbanas durante el verano cuando las temperaturas de la superficie del océano están más frías que las de la tierra y el viento sopla hacia tierra firme. Donde las ciudades están 
rodeadas por superficies rurales mojadas, el enfriamiento más lento de estas superficies puede reducir las magnitudes de la isla de calor, especialmente en climas cálidos y húmedos (OKE et al., 1991).

Figura 5 - Ilustra la Isla de Calor: en color rojo se puede observar las islas de calor más notables en la ciudad Madrid

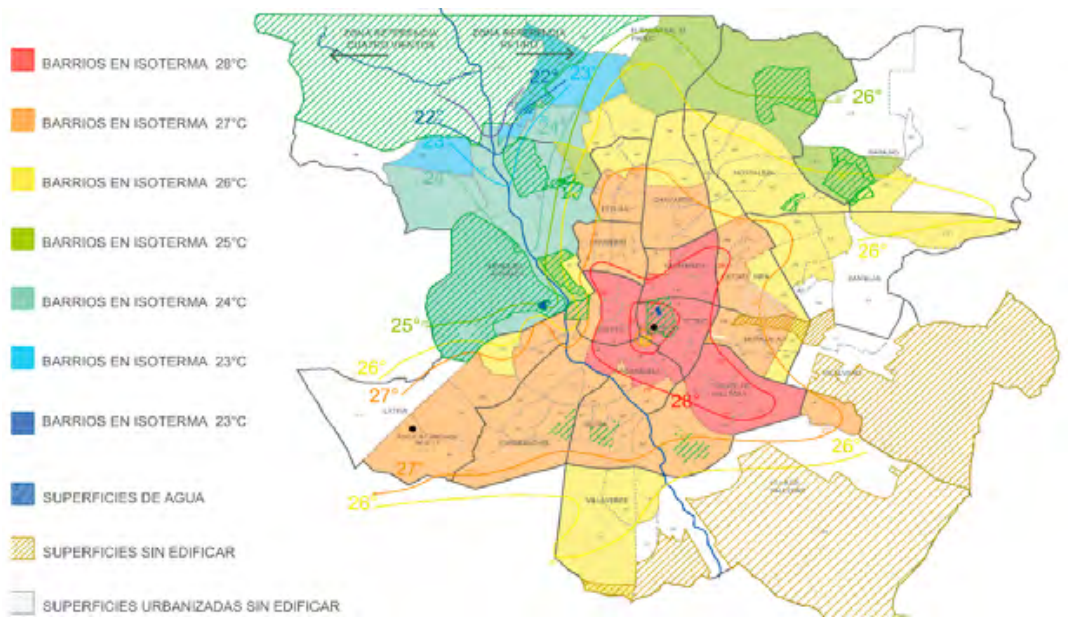

Fuente: investigadores de la Universidad Politécnica de Madrid (UPM).

Este efecto se debe a la sustitución de la vegetación por edificios y calles, lo que tiene como consecuencia una mayor absorción de radiación solar por parte de las superficies construidas y pavimentadas, y que, junto a otros factores antropogénicos, emiten calor al aire circundante. De donde se produce un aumento de la temperatura local (ver Figuras 5-6), dando lugar a un microclima urbano que afecta al comportamiento energético de las edificaciones.

Por lo que está muy claro las variaciones que está sufriendo el medio ambiente, por las consecuencias del cambio climático, derivado de la tala de árboles, edificaciones urbanísticas y la no preservación del medio ambiente. Por lo que todo esto rompe el equilibrio medio ambiental, provocando un aumento en los de los gases nocivos para la salud, sus niveles de contaminación permitidos. 
Figura 6 - La Isla de Calor en una ciudad modelo, donde las temperaturas son generalmente más bajas en las fronteras urbanas-rurales que en los densos centros de las ciudades

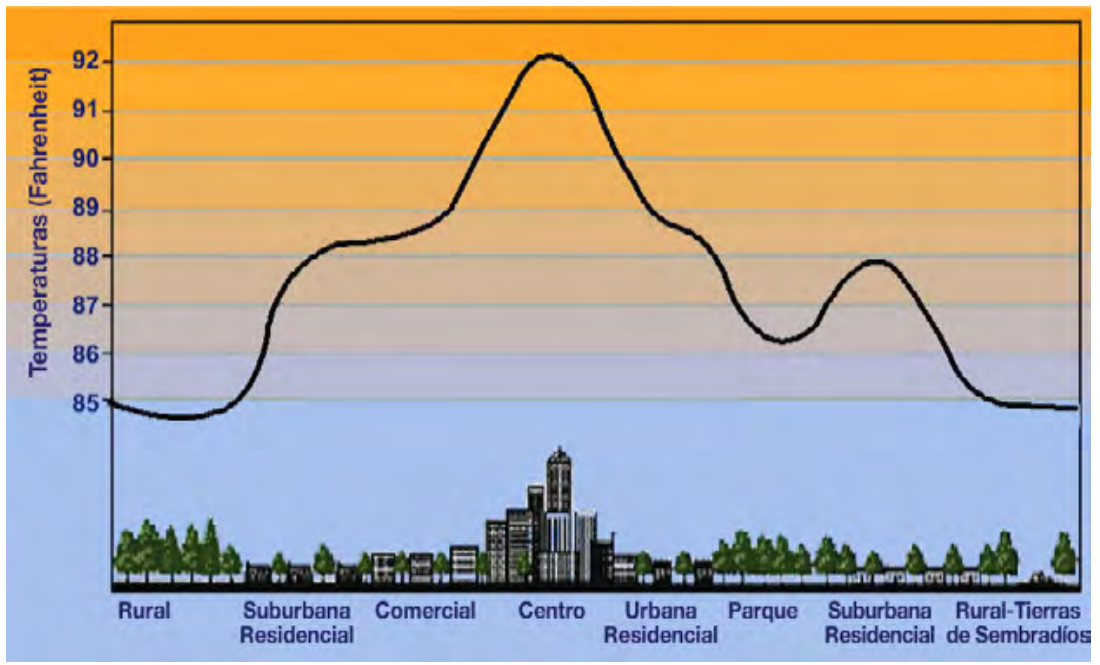

Fuente: GARDINER, 2009.

\section{Conclusiones}

Aquí podemos concluir, sobre la importancia de la conservación del medio ambiente y del buen cumplimiento de las normas legislativas para un mejor aire limpio y libre de contaminación en las ciudades. También la importancia de los estudios científicos en relación a los contaminantes con la salud. Por último antes de edificar la ciudad hay que hacer un estudio para no crear islas de calor que influyen negativamente en el medio ambiente.

\section{Referencias}

EEA: Air quality in larger cities in the European Union A contribution to the Auto-Oil II programme. (Aalst, R. Coord.) European Environment Agency. Topic report 3/2001 55 pp. 2001a. 
EEA (2002.1): Air quality in Europe state and trends 1990-99. (Aalst, R. Coord.). European Environment Agency. Topic report 2/2002. 83 p. 2001 b.

FENGER, Jes, Urban air quality. Atmospheric Environment, 33, p. 4.877-4.900, 1999.

FERNÁNDEZ-GARCIA, Felipe. El clima urbano: aspectos generales y su aplicación en el área de Madrid, 2012.

GARDINER, Lisa. Ventanas al Universo, de acuerdo a una figura del Laboratorio Nacional de Lawrence Berkeley. 2009. Disponível em: <http://www.windows2universe. org/earth/Atmosphere/urban_heat.html\&lang=sp >.

GUENTHER, Alex et al. A global model of natural volatile organic compound emissions. Journal of Geophysical Research, 100, p. 8.873-8.892, 1995.

JOL, A.; AALST, R. (Coord). EEA. (2002.2): Air pollution by ozone in Europe summer 2002. Overview of exceedances of EC ozone threshold values during the summer season April-August 2002. European Environment Agency. Topic report, 2/2002, 23 p., 2002.

LINARES GIL, Cristina; DÍAZ JIMÉNEZ, J. Las PM2.5 y su impacto sobre la salud. El caso de la ciudad de Madrid. Ecosostenible, Centro Nacional de Epidemiología, Escuela Nacional de Sanidad, Instituto de Salud Carlos III, n. 35, p. 32-37, 2008.

LÓPEZ BONILLA, Diego. El medio ambiente. Madrid: Cátedra, 1994. 395 p.

NASA's Aqua satellite captured this image of the clouds over Canada. Entwined within the clouds is the smoke billowing up from the wildfires that are currently burning across a large expanse of the country. The smoke has become entrained within the clouds causing it to twist within the circular motion of the clouds and wind. This image was taken by the Moderate Resolution Imaging Spectroradiometer (Modis) instrument on the Aqua satellite on May 9, 2016.

OKE, Tim et al. Simulation of surface urban heat islands under "ideal" conditions at night. Part 2: Diagnosis of causation. Boundary-Layer Meteorology, 56, p. 339-358, 1991.

RUDD, J. Howard. Emissions of volatile organic compounds from stationary sources in the United Kingdom: speciation. AEA Technology Report, AEA/CS/REMA-029. AEA Technology. Oxfordshire, UK, 1995.

WHITTAKER, Andy et al. Killer smog of London, 50 years on: particle properties and oxidative capacity. Science of the total Environment, p. 334-335, 435-445, 2004.

WICHMANN, H.-Erich, 20 years alter the winter smog episode. The particle problem then and today. International Journal of Environment and Health, 20, p. 489-491, 1985.

WORLD HEALTH ORGANIZATION. WHO. Air Quality Guidelines for Europe. 2. ed. Who Regional Publications, European Series, n. 91. Copenhagen, 2000a. 
WORLD HEALTH ORGANIZATION. WHO. Environmental Health Indicators: Development of a methodology for the WHO European Region. Interim report 6 november 2000 (EUR/00/5026344), 2000b.

Environmental Health Indicators for the WHO European Region. Update of methodology. World Health Organization Regional office Interim for Europe. May 2002, (EUR/02/5039762), 2002.

. Environmental Health Indicators for Europe. A pilot indicator based report. World Health Organization for European Region. Junio, 2004. 58 p.

Recebido em: 9/6/2016

Aceito em: 15/12/2016 